\title{
Persistent hiccups
}

\section{If excluding or treating any underlying pathology fails try chlorpromazine}

Hiccups consist of brief bursts of intense inspiratory activity, involving the diaphragm and inspiratory intercostal muscles, with reciprocal inhibition of the expiratory intercostal muscles. Glottic closure occurs almost immediately after the onset of diaphragmatic contraction, thus minimising the ventilatory effect as well as generating the characteristic sound and sense of discomfort. ${ }^{1}$ Often associated with gastric distension, sudden changes in temperature, emotion, or ingestion of alcohol, ${ }^{2}$ hiccups usually resolve spontaneously or with simple measures. Hiccups continuing for longer than 24 hours are rare and may indicate serious underlying disease; they may also produce deleterious effects such as postoperative wound dehiscence or infection, fatigue, dehydration, weight loss, and, in extreme cases, death. ${ }^{3}$

Intractable hiccups may result from structural or functional disturbances of the medulla (affecting the region of the vagal nuclei and the nucleus tractus solitarius ${ }^{4}$ ) or of afferent or efferent nerves to the respiratory muscles. Metabolic and endocrine disorders, drugs, general anaesthesia and psychogenic problems may also cause them. Hiccups are particularly associated with structural lesions of the medulla including infarction in the territory of the posterior inferior cerebellar artery, tumour, ${ }^{5}$ tuberculoma ${ }^{6}$ abscess, ${ }^{7}$ syrinx, haematoma, and demyelination. ${ }^{8}$ They may also be a feature of infections of the central nervous system such as viral encephalitis and, less commonly, meningitis, encephalitis lethargica, syphilis, ${ }^{9}$ and HIV encephalopathy. Neurogenic hiccups indicate involvement of the medullary region intimately associated with respiratory control. Their occurrence may anticipate the development of irregularities of the respiratory rhythm culminating in respiratory arrest. ${ }^{10}$ Neurogenic hiccup, however, rarely occurs in isolation, and associated brainstem or long tract signs are usually evident.

Disorders of the oesophagus and other parts of the gastrointestinal tract are occasionally associated with protracted hiccups due to stimulation of the visceral afferent fibres of the vagus nerve. In particular, hiccups occur with gastrooesophageal reflux, achalasia, gastric distension, oesophageal or small bowel obstruction, and pancreatic or biliary disease. ${ }^{211}$ Similarly, involvement of the auricular branch of the vagus may explain the association of hiccups with a foreign body in the external auditory meatus. ${ }^{12}$ By a similar mechanism, mediastinal disease, including tumours and thoracic aortic aneurysms, may cause hiccups by affecting the thoracic afferent fibres. ${ }^{13}$ Protracted hiccups may also be associated with diaphragmatic irritation caused by sub- phrenic and hepatic disease, ${ }^{9}$ pleural or pericardial effusion, and lateral myocardial infarction ${ }^{14}$ - possibly due to involvement of efferent phrenic nerve fibres.

Hiccups are common during light anaesthesia and may interfere with both the surgical procedure and the efficiency of ventilation. They are also associated with systemic disorders including diabetes mellitus, uraemia, hypocalcaemia, hyponatraemia, ${ }^{2}$ and Addison's disease. ${ }^{15}$ Iatrogenic causes include short acting barbiturates, methyldopa, and steroids. It has been suggested that protracted hiccups in women are commonly psychogenic, ${ }^{9}$ but this seems unlikely, particularly when hiccups persist during sleep.

The more than 100 forms of physical or pharmacological treatment for intractable hiccups include prayers to St Jude, the patron saint of lost causes. ${ }^{16}$ The vast array of anecdotal and uncontrolled reports reflects both the self limiting nature of hiccups and the lack of any consistently satisfactory and reliable treatment.

The first line of management is to reverse or treat any underlying cause, including relieving oesophageal obstruction or gastric distension. Raising carbon dioxide pressure reduces hiccup frequency, and reducing this pressure increases their amplitude. ${ }^{1}$ This may explain why breath holding, rebreathing, and other techniques designed to interrupt the respiratory rhythm are effective. If the patient has a tracheostomy bypassing the glottis, however, the vigorous and uninterrupted inspiratory spasm may produce hyperventilation and thereby exacerbate the hiccups. ${ }^{17}$

Stimulation of the pharynx reliably inhibits hiccups, although the effect may be only temporary. Salem et al described using frictional movement of a catheter sited in the pharynx opposite C2 and C3. ${ }^{18}$ Presumably similar pharyngeal stimulation is achieved by sipping iced water, gargling, swallowing granulated sugar, and various other manipulations of the uvula or nasopharynx. Hiccups may also be inhibited by stimulating other parts of the upper respiratory tract and external auditory meatus. ${ }^{2}$

Modulating phrenic nerve function by transection, anaesthetic block, or (electrical or mechanical) stimulation is often unsuccessful because associated inspiratory bursts persist in intercostal and accessory respiratory muscles. ${ }^{1}$ Furthermore, blocking both phrenic nerves causes complete diaphragmatic paralysis and may precipitate severe respiratory impairment.

Many drugs have been used to treat prolonged hiccups resistant to physical methods. Chlorpromazine remains the 
most consistently effective agent, especially when given as a single intravenous bolus, but haloperidol is also valuable. ${ }^{19}$ Other drugs, including metoclopramide, clonazepam, and antiepileptics (carbamazepine, ${ }^{8}$ valproic acid, ${ }^{20}$ and phenytoin), may be of value, particularly in neurogenic hiccups. More recent reports have described resolution of prolonged hiccups with amitriptyline, ${ }^{21}$ nifedipine, ${ }^{22}$ amantidine, ${ }^{23}$ and baclofen. ${ }^{24}$ Classes of agents that have been beneficial in single cases or short reports include tricyclic antidepressants, anticholinergics, benzodiazepines, stimulants of the central nervous system, $\mathrm{H}_{2}$ receptor antagonists, and dopamine agonists.

When faced with a patient with intractable hiccups it is necessary to exclude or treat any underlying disease. Then, if simple physical measures fail to abort the attack and the hiccups last for longer than 24 hours, treatment with chlorpromazine, metoclopramide, antiepileptics, or baclofen should be considered.

ROBIN S HOWARD

Consultant Neurologist,

St Thomas's Hospital,

London SE1 7EH
3 Kolodzik PW, Eilers MA. Hiccup (singulutus): review and approach to management. Ann Emerg Med 1991;20:565-73.

4 Newsom Davis J. Pathological interoceptive responses in respiratory muscles and the mechanism of hiccup. In: Desmedt J, ed. New developments in EMG and clinical neurophysiology. Vol 3. Basel: Karger, 1973:751-60.

5 Stotka VL, Barcay SJ, Bell HS, Clare FB. Intractable hiccough as the primary manifestation of brain stem tumor. Am f Med 1962;32:13-5.

6 Al Deeb SM, Sharif H, Al Moutaery K, Biary N. Intractable hiccup induced by brainstem lesion. f Neurol Sci 1991;103:144-50.

7 Jansen PHP, Joosten EMG, Vingerhoets HM. Persistent periodic hiccups following brain abscess: a case report. I Neurol Neurosurg Psychiatry 1990;53:83-4.

$8 \mathrm{McF}$ arling DA, Susac JO. Hoquet diabolique: intractable hiccups as a manifestation of multiple sclerosis. Neurology 1979;29:797-801.

9 Soudjian JV, Cain JC. Intractable hiccup. Postgrad Med 1968;43:72-7.

10 Howard RS, Wiles CM, Hirsch NP, Loh L, Spencer GT, Newsom Davis J. Respiratory involvement in multiple sclerosis. Brain 1992;115:479-94.

11 Kaufman HJ. Hiccups: an occasional sign of oesophageal obstruction. Gastroenterology 1982;82: 1443-5.

12 Lossos IS, Beuer R. A rare cause of hiccups. N Engl F Med 1988;318:711.

13 Samuels L. Hiccup: a ten year review of anatomy, etiology and treatment. Can Med Assoc $f$ 1952:67:315-22.

14 Ikram H, Orchard RT, Read SEC. Intractable hiccuping in acute myocardial infarction. $B M 7$ 1971;ii:504.

15 Hardo PG. Intractable hiccups-an early feature of Addison's disease. Postgrad Med 7 1989;65: 918-9.

16 McWhirter N, McWhirter R. Guinness book of records. New York: Sterling, 1974:41

17 Plum F, Posner JB. The diagnosis of stupor and coma. 3rd ed. Philadelphia: F A Davis, 1982:39.

18 Salem MR, Baraka A, Rattenborg CC, Holaday DA. Treatment of hiccups by pharyngeal stimulation in anesthetized and conscious subject. $\Im A M A$ 1967;202:126-30.

19 Williamson BWA, Macintyre IMC. Management of intractable hiccup. BMF 1977;ii:501-3.

20 Jacobson PL, Messenheimer JA, Farmer TW. Treatment of intractable hiccups with valproic acid. Neurology 1981;31:1458-60

21 Stanlnkowicz R, Fich A, Troudart T. Amitriptyline for intractable hiccups. $N$ Engl f Med 1986:315:64-5.

22 Lipps DC, Jabbari B, Mitchell MH, Daigh JD. Nifedipine for intractable hiccups. Neurology 1990;40:531-2.

23 Askenasy JJM, Boiangiu M, Davidovitch S. Persistent hiccup cured by amantidine. $N$ Engl f Med 1988;318:711.

24 Burke AM, White AB, Brill N. Baclofen for intractable hiccups. N Engl F Med 1988;319:1354

\section{Driving and diabetes}

\section{By not notifying the licensing authority many diabetic drivers are breaking the law}

Medical disabilities are thought to be responsible for very few road traffic accidents, but diabetes is designated a prospective disability because of its progressive severity, the development of complications, and the potential side effects of treatment. Although diabetic drivers are legally required to declare their diabetes to the Driver and Vehicle Licensing Agency, it has been known for over a decade that many do not. ${ }^{1-3}$ Most diabetic drivers are unaware that failure of notification is regarded as concealment of a material fact, and motor insurers can repudiate liability in the event of a claim.

The survey in general practice reported on p 1265 by Saunders suggests that at least a quarter of all diabetic drivers still fail to notify the regulatory authority. ${ }^{4}$ This compares with recent surveys of insulin treated diabetic drivers attending specialist centres in Belfast and Edinburgh, where the rates of failure to notify the licensing agency were $41 \%{ }^{5}$ and $34 \%$ respectively. This failure of notification may represent ignorance of the law or deliberate concealment because of fear that the licence may be revoked on medical grounds. Although the onus to declare rests with the individual driver, doctors providing diabetic care, who include general practitioners, are responsible for informing patients of this legal requirement and for offering practical advice. ${ }^{7}$ This should be included in the education of newly diagnosed diabetic patients.

The principal problems that may interfere with ability to drive are hypoglycaemia and visual impairment. Insulin induced hypoglycaemia of varying severity has been reported during driving by one third of insulin treated diabetic drivers ${ }^{156}$ and is known to cause road traffic accidents. ${ }^{1568}$ Appreciable impairment of driving skills during controlled hypoglycaemia has been shown with a sophisticated driving simulator, ${ }^{9}$ and hypoglycaemia need not be severe to interfere with driving ability. Neuroglycopenia can induce cognitive dysfunction without provoking a symptomatic response or affecting the conscious level, ${ }^{10}$ and hypoglycaemia induced automatism, associated with irrational and compulsive behaviour while driving, has been reported.'

Inability to perceive the onset of hypoglycaemia through reduced intensity or loss of warning symptoms poses a serious problem for diabetic drivers and is a common reason for revocation of their licence. The blood glucose threshold at which autonomic warning symptoms start can be altered during strict glycaemic control ${ }^{11}$ but is restored when control is relaxed. A chronic form of "hypoglycaemia unawareness" is present to some degree in half of patients with insulin dependent diabetes of more than 15 years' duration. ${ }^{12}$ This acquired and irreversible complication is associated with an increased frequency of severe hypoglycaemia ${ }^{12}$ but is not an absolute contraindication to driving if the affected person can show prolonged freedom from hypoglycaemia with frequent self monitoring of the blood glucose concentration.

Because patients whose diabetes is controlled by diet are not exposed to hypoglycaemia routine notification to the licensing agency is no longer required and their driving licences are not restricted. These patients, however, do not have "mild" diabetes, as established retinopathy is present at diagnosis in a fifth of patients and all are susceptible to diabetic complications such as maculopathy, which can seriously reduce visual acuity. Previous surveys identified very few diabetic drivers who had inadequate vision for driving. ${ }^{5}$ A review of 17543 drivers with non insulin dependent diabetes, by the medical advisory branch of the Driver and Vehicle Licensing Agency, showed that the driving licence had been refused or revoked in only 41 cases $(0.23 \%)$, mostly because of impaired vision (Elizabeth Rowse, personal communication). Although these figures suggest that visual impairment is rare among non-insulin dependent diabetic 